\title{
Thrombotic thrombocytopenic purpura: An unusual presentation with intracranial bleed
}

\author{
Shaik Mohammad Tahaseen', Ravi Kirti², Subhash Kumar ${ }^{3}$ \\ From ${ }^{1}$ Post Graduate Trainee, ${ }^{2}$ Additional Professor and Head, Department of General Medicine, ${ }^{3}$ Additional Professor, Department of Radiodiagnosis, \\ AIIMS, Patna, Bihar, India
}

\begin{abstract}
Thrombotic thrombocytopenic purpura (TTP) is a rare and life-threatening thrombotic microangiopathy characterized by microangiopathic hemolytic anemia, severe thrombocytopenia, and organ ischemia linked to disseminated microvascular platelet-rich thrombi. We present the case of a 44-year-old lady who presented with severe thrombocytopenia and anemia that did not respond to repeated transfusions and steroids. Non-contrast computed tomography scan of the brain revealed an intracranial bleed. Schistocytes were seen on the peripheral blood smear. A provisional diagnosis of TTP was made. Plasmapheresis could not be done due to her deteriorating hemodynamic status. She succumbed to her illness in spite of the best possible efforts. This case highlights the need for keeping a high index of suspicion for TTP as early diagnosis and prompt initiation of plasmapheresis are crucial for preventing death.
\end{abstract}

Key words: Thrombotic thrombocytopenic purpura, Microangiopathic hemolytic anemia, ADAMTS-13, Plasma exchange therapy

$\mathrm{T}$ hrombotic thrombocytopenic purpura (TTP) is a rare and life-threatening thrombotic microangiopathy (TMA) characterized by microangiopathic hemolytic anemia (MAHA), severe thrombocytopenia, and organ ischemia linked to disseminated microvascular platelet-rich thrombi. The condition is caused by either hereditary or acquired severe deficiency of ADAMTS-13 required for cleaving large multimers of von Willebrand factor (VWF) attached to the endothelial surfaces of blood vessels that are responsible for the formation of plateletrich thrombi [1]. Acquired cases are due to autoantibodies against ADAMST-13. Early diagnosis and prompt initiation of management are very crucial. Untreated, the condition has a mortality of about $95 \%$. However, with prompt treatment with plasmapheresis, the mortality rate may be reduced to $<80 \%$ [2].

We describe here an unusual case that presented with an intracranial bleed.

\section{CASE REPORT}

A 44-year-old female presented to a tertiary care hospital with the chief complaints of reddish spots all over the body for 14 days. She had also noticed bleeding from her gums over the past 8 days. There was a history of scant vaginal bleeding unrelated to

\section{Access this article online}

Received - 02 August 2021

Initial Review - 18 August 2021

Accepted - 25 September 2021

DOI: $10.32677 /$ ijcr.v7i10.3059 menstruation for 3 days. There was no history of bleeding from any other site. She had been drowsy and confused for 1 day at the time of admission. There was no history of fever. Medical history was unremarkable apart from an episode of scrub typhus 4 months ago which was treated with doxycycline. There was no history of menorrhagia. She had three children, the youngest being 4-year-old. There was no history of abortion or stillbirth.

On presentation, the patient was drowsy and confused with a Glasgow Coma Scale (GCS) of 13 (E3V4M6). She had a blood pressure of $122 / 80 \mathrm{mmHg}$, heart rate of 80 beats/min, respiratory rate of $20 / \mathrm{min}$, and oxygen saturation of $100 \%$ on room air. She was afebrile. General examination revealed moderate to severe pallor but no icterus, cyanosis, clubbing, lymphadenopathy, or edema. Multiple petechial and ecchymotic patches were present over the face, both upper and lower limbs, neck, and abdomen. A detailed neurological examination was difficult. However, she was moving all four limbs. There was no neck rigidity. Both pupils were dilated and sluggishly reacting to light. On inspection, muscle bulk was normal throughout. The tone was reduced in the left lower limb. She had an extensor plantar response on the left side and a withdrawal response on the right side. Cardiorespiratory and abdominal examination was normal.

Her capillary blood glucose was $372 \mathrm{mg} / \mathrm{dL}$. Arterial blood gas was normal. Initial blood tests revealed hemoglobin $5.5 \mathrm{~g} /$ $\mathrm{dL}$, white blood count $18 \times 10^{9} / \mathrm{L}$, platelet count $15000 / \mathrm{mm}^{3}$,

Correspondence to: DR. Shaik Mohammad Tahaseen, Department of General Medicine, AIIMS Patna, Phulwarisharif, Patna - 801 507, Bihar, India. E-mail: smtahaseen@gmail.com

(C) 2021 Creative Commons Attribution-NonCommercial 4.0 International License (CC BY-NC-ND 4.0). 


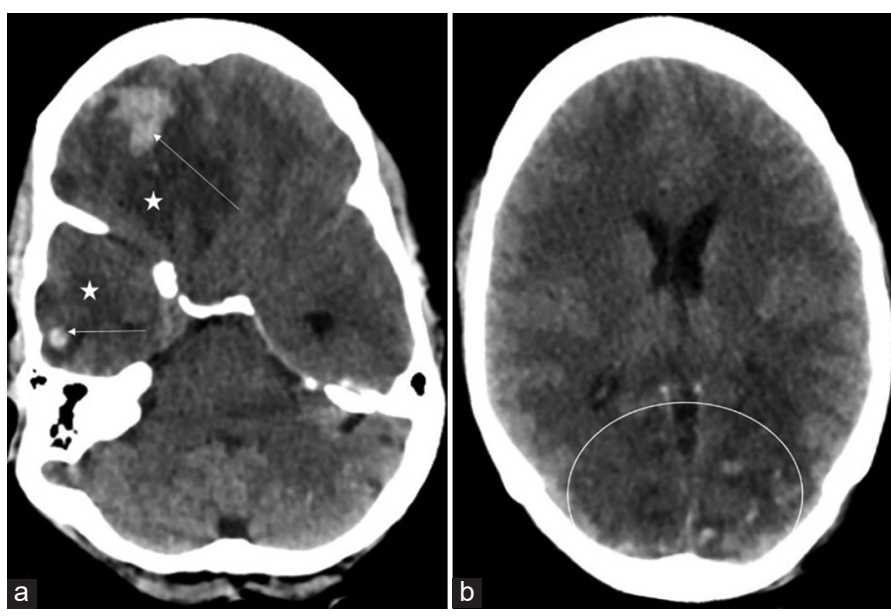

Figure 1: Computed tomography scan images (a) axial section showing hemorrhages in right frontal and temporal lobes (white arrow) with surrounding edema (star) (b) Axial section of scan performed 6 days later showing patchy hyperintensities suggestive of hemorrhagic foci within areas of hypodensity suggesting edema, in both parieto-occipital regions (white oval)

C-reactive protein $53 \mathrm{mg} / \mathrm{dL}$, prothrombin time $12.8 \mathrm{~s}$, urea $23.5 \mathrm{mg} / \mathrm{dl}$, and creatinine $0.61 \mathrm{mg} / \mathrm{dl}$. Non-contrast computed tomography (NCCT) brain showed a hemorrhagic contusion in the right basi-frontal region with mass effect, midline shift, and uncal herniation (Fig. 1a).

Four units of platelets were transfused. The patient was kept nil per oral till further advice. Intravenous fluid and injection regular insulin according to the sliding scale were started. Injection tranexamic acid $500 \mathrm{mg}$ intravenous thrice daily and injection ceftriaxone $2 \mathrm{~g}$ intravenous once daily were prescribed. Injection mannitol $200 \mathrm{ml}$ intravenous stat was given followed by $100 \mathrm{ml}$ intravenous 4 times a day. Urgent neurosurgery referral was done and the patient was shifted to the medical intensive care unit. The guarded prognosis was explained to her relatives.

Over the next few days, her condition deteriorated further. GCS fell to 8 (E1V2M5). She had multiple episodes of fever necessitating several doses of injection paracetamol $1 \mathrm{~g}$ intravenously. She was tachycardic (141 beats/min) and tachypnoeic (44 cycles/min). Injection methylprednisolone $500 \mathrm{mg}$ intravenous once daily for 3 days was started on the advice of a hematologist in view of the possible diagnosis of immune thrombocytopenic purpura. Repeat blood tests revealed a hemoglobin of $4.9 \mathrm{~g} / \mathrm{dL}$, platelet $7000 / \mathrm{mm}^{3}$, and white blood count of $21.2 \times 10^{9} / \mathrm{L}$. Tests for scrub typhus, dengue, and malaria came back as negative. One unit packed red blood cells and one unit single donor platelets were transfused. On further blood tests, lactate dehydrogenase (LDH) was increased up to $1449 \mathrm{U} / \mathrm{L}$, ferritin was normal at $44.7 \mathrm{ng} / \mathrm{ml}$, D-dimer was increased at $1.52 \mathrm{mg} / \mathrm{L}$, fibrinogen was normal at $220 \mathrm{mg} / \mathrm{dl}$, direct Coomb's test was negative, peripheral blood smears revealed schistocytes, and the PLASMIC SCORE was 7. Escherichia coli was grown on urine culture. Injection amikacin $750 \mathrm{mg}$ intravenously was started according to the sensitivity report. However, her hemoglobin and platelet count continued to fall repeatedly even as her total WBC count kept climbing up. In view of her falling
GCS and persistent fever, injection meropenem $1 \mathrm{~g}$ intravenous thrice a day was advised, and amikacin was stopped.

A repeat NCCT revealed new subarachnoid hemorrhage $(\mathrm{SAH})$ in the left occipital region and hemorrhagic foci within areas of hypodensity suggesting edema in both parieto-occipital regions (Fig. 1b). The case was discussed again with the consultant hematologist. In view of elevated LDH, fever, and fluctuating neurological course, a provisional diagnosis of TTP was made. Plasmapheresis (1.5 L volume) was planned. If this was not possible, it was planned to transfuse 4 units of fresh frozen plasma every day.

However, that night, the patient deteriorated further with hypoxia, hypotension, and labored breathing requiring intubation, mechanical ventilation, and inotropic support. Tablet prednisolone $50 \mathrm{mg}$ daily was advised and rituximab $375 \mathrm{mg} / \mathrm{m}^{2}$ infusion once weekly was started. However, she developed oliguria and severe metabolic acidosis. Her urea and creatinine were $102.6 \mathrm{mg} / \mathrm{dl}$ and $1.22 \mathrm{mg} / \mathrm{dl}$, respectively. She had a cardiac arrest from which she was revived with a return of spontaneous circulation. She received one session of hemodialysis. Unfortunately, she had another cardiac arrest from which she could not be revived.

\section{DISCUSSION}

TTP is a rare, life-threatening disease with an incidence of approximately 2/million/year [3]. It is a rare form of TMA characterized by a pentad of MAHA, severe thrombocytopenia, acute kidney injury, fever, and neurological symptoms [4]. There is ischemic end-organ damage resulting from the formation of platelet-rich thrombi in the microvasculature [3]. TTP is distinguished from other TMAs by severe deficiency of ADAMTS-13 (a disintegrin and metalloproteinase with a thrombospondin type 1 motif, member 13), a plasma protein that cleaves VWF multimers. Without the proteolytic activity of ADAMTS13, the uncleaved ultra-large VWF multimers accumulate and induce excessive platelet adhesion and aggregation, leading to the formation of disseminated micro thrombosis and clinical features of TTP [4].

In patients with congenital TTP, severe ADAMTS13 deficiency results from a biallelic mutation in the ADAMTS13 gene with severe ADAMTS13 deficiency (activity <10\%), whereas, immune-mediated TTP is associated with anti-ADAMTS13 autoantibodies, which neutralize or induce clearance of the ADAMTS13 protein $[3,5]$. Immune TTP may occur in isolation. This is called primary TTP. It may also occur in association with a predisposing condition such as Systemic Lupus Erythematosus, malignancy, HIV, pregnancy, and the use of certain drugs (secondary TTP).

Platelet count lower than $20000 / \mathrm{mm}^{3}$, a positive antiADAMTS-13 antibody titer, and the presence of gastrointestinal symptoms are predictive of primary TTP, whereas, severe anemia (hemoglobin $<7 / \mathrm{dl}$ ), and the presence of fever are associated with secondary TTP. Neurological involvement is more common and more severe in primary TTP with a greater percentage of cases presenting with stroke and coma [3]. 
This patient presented with petechiae, bleeding gums, and bleeding per vagina that have been reported in $46 \%$ of the patients [3]. Some of the main features of TTP such as MAHA and thrombocytopenia were present in her case. She also had features of sepsis with multiple episodes of fever. This can be found in secondary types of immune TTP [3]. Acute kidney injury is another characteristic feature of TTP $[3,4]$. Although initially, this patient did not have renal failure but at a later stage, she developed severe renal failure with anuria for which dialysis was carried out but without any benefit. Forty to fifty percent of the patients develop neurological deterioration $[3,4]$. Our patient had a fluctuating sensorium. NCCT brain revealed a right frontal hemorrhagic stroke. A repeat NCCT brain 6 days later also revealed a left occipital SAH. Both these NCCT findings are very unusual as commonly ischemic stroke is seen with TTP.

The PLASMIC score predicts ADAMTS-13 deficiency in suspected TTP with high discrimination. The presence of schistocytes on peripheral blood smear should be considered a precondition for applying the score. The score gives one point each for the following features: Platelet count $<30,000 / \mathrm{mL}$, hemolysis (defined by reticulocyte count $>2.5 \%$, undetectable haptoglobin, or indirect bilirubin $>2 \mathrm{mg} / \mathrm{dL}$ ), absence of active cancer, absence of a history of solid organ or stem cell transplant, mean corpuscular volume $<90 \mathrm{fL}, \mathrm{INR}<1.5$, and creatinine $<2.0 \mathrm{mg} / \mathrm{dL}$. A score of four or less predicts a low probability, five an intermediate probability, and a six or seven a high probability of TTP [6]. This patient had a PLASMIC score of seven.

Initially, ITP was suspected but the clinical course and failure to respond to pulse steroid therapy prompted the consideration of an alternative diagnosis. Furthermore, macrophage activation syndrome and DIC were ruled out in view of elevated LDH, normal ferritin, fibrinogen, and PT/INR. The mainstay of treatment of TTP is with plasma exchange along with steroids as early as possible $[3,4]$. Due to severe hemodynamic compromise by the time a provisional diagnosis of TTP was made, plasma exchange was not possible. Early treatment with Rituximab $375 \mathrm{mg} / \mathrm{m}^{2}$ once a week for 1 month has been found to be beneficial [7,8]. Unfortunately, it did not change the outcome in this case.

\section{CONCLUSION}

As TTP is a rare disease with potentially serious complications, early and proper diagnosis, and initiation of appropriate and timely treatment can be life-saving. Although ischemic stroke is commonly seen with TTP, the diagnosis should still be considered in the case of intracranial bleeding if other features of TTP are present.

\section{REFERENCES}

1. Joly BS, Coppo P, Veyradier A. Thrombotic thrombocytopenic purpura. Blood 2017;129:2836-46.

2. Hovinga K, Coppo P, Lämmle B, Moake JL, Miyata T, Vanhoorelbeke K. Thrombotic thrombocytopenic purpura. Nat Rev Dis Primers 2017;3:1-17.

3. Chiasakul T, Cuker A. Clinical and laboratory diagnosis of TTP: An integrated approach. Hematology Am Soc Hematol Educ Program 2018;2018:530-8.

4. Sugarman R, Tufano AM, Liu JM. Large vessel stroke as initial presentation of thrombotic thrombocytopenic purpura. BMJ Case Rep 2018;2018:bcr2017221857.

5. Borogovac A, George JN. Stroke and myocardial infarction in hereditary thrombotic thrombocytopenic purpura: Similarities to sickle cell anemia. Blood Adv 2019;3:3973-6.

6. Bendapudi PK, Li A, Hamdan A, Fry AM, Uhl L, Marques M, et al. Derivation and prospective validation of a predictive score for the rapid diagnosis of thrombotic thrombocytopenic purpura: The plasmic score. Blood 2014;124:231.

7. Lim W, Vesely SK, George JN. The role of rituximab in the management of patients with acquired thrombotic thrombocytopenic purpura. Blood 2015;125:1526-31.

8. Illner N, Wolf G. Rituximab as effective therapy in very severe thrombotic thrombocytopenic purpura (TTP). Dtsch Med Wochenschr 2010;135:71-4.

Funding: None; Conflicts of Interest: None Stated.

How to cite this article: Tahaseen SM, Kirti R, Kumar S. Thrombotic thrombocytopenic purpura: An unusual presentation with intracranial bleed. Indian J Case Reports. 2021;7(10):436-438. 